Research Article

\title{
Limit Properties of the Largest Entries of High-Dimensional Sample Covariance and Correlation Matrices
}

\author{
Xue Ding \\ School of Mathematics, Jilin University, Changchun 130000, China \\ Correspondence should be addressed to Xue Ding; dingxue83@jlu.edu.cn \\ Received 30 April 2021; Accepted 15 September 2021; Published 29 September 2021 \\ Academic Editor: Jing-Feng Tian \\ Copyright (c) 2021 Xue Ding. This is an open access article distributed under the Creative Commons Attribution License, which \\ permits unrestricted use, distribution, and reproduction in any medium, provided the original work is properly cited. \\ In this paper, we consider the limit properties of the largest entries of sample covariance matrices and the sample correlation \\ matrices. In order to make the statistics based on the largest entries of the sample covariance matrices and the sample correlation \\ matrices more applicable in high-dimensional tests, the identically distributed assumption of population is removed. Under some \\ moment's assumption of the underlying distribution, we obtain that the almost surely limit and asymptotical distribution of the \\ extreme statistics as both the dimension $p$ and sample size $n$ tend to infinity.
}

\section{Introduction}

The sample covariance matrices play an important role in multivariate statistical inference. Its limiting properties are fundamental in hypothesis testing, principal component analysis, factor analysis, and discrimination analysis. The sample correlation matrix is another popular random matrix that plays an important role in independence test. Under classical statistical settings, where the number of variables is fixed, sample covariance matrix and correlation matrix perform well. However, in modern applications, the dimension of data is often high. Under such high-dimensional settings, sample covariance matrices and correlation matrices become inaccurate due to "curse of dimensionality." Despite their extensive use in practice, under high-dimensional assumption, there are many theoretical understandings of spectral properties of sample covariance under the high-dimensional setting where the number of samples and variables both grow large at the same rate, see $[1,2]$. The sample correlation matrices have many same spectral properties as sample covariance matrices under some assumptions, such as almost surely limit of extreme eigenvalue and the convergence of empirical eigenvalue distribution, see [3]. However, the CLTs for linear spectral statistics is different, see $[4,5]$.
The study of the largest entries of sample correlation matrices is important for independence test. As both dimension $p$ and sample size $n$ tend to infinity, for i.i.d. random variable Jiang [6] prove that the asymptotical distribution is extreme distribution of type I. Later, Li and Rosalsky [7] and Li et al. [8, 9] relax that moment assumptions. Li and Xue [10] and Li et al. [11] studied the asymptotic joint distribution of the maximum test statistic and the quadratic test statistic for one sample covariance test. Recently, Yu et al. [12] provide a new perspective to exploit the full potential of quadratic form statistics and maximum form statistics for testing high-dimensional covariance matrices. The extreme-value form statistic also can be used in Zheng et al. [13] to establish testing for correlation structures under high-dimensional setting. These studies related to the largest entries of sample correlation matrices make it more interesting to investigate the asymptotic distribution of the largest entries of sample covariance and correlation matrices. The i.i.d. assumption is usually needed in the literature. Liu and Lin [14] relax the independent assumption but require the underlying sequence to be the strictly stationary. In this paper, we remove the identically distributed condition and established limit and asymptotic distribution of the largest entries of sample covariance and correlation matrices. The rest of the paper is organized as 
follows. In Section 2, we give the main results; in Section 3, we first present some useful lemmas and then give the proofs of the main results.

\section{Main Results}

Suppose that $\mathbf{x}_{1}, \mathbf{x}_{2}, \ldots, \mathbf{x}_{n}$ are $n$ independent observations of the $p$-dimensional real-valued random vector $\mathbf{x}$. Assume that $\mathbf{x}_{j}=\left(x_{i j}, 1 \leq i \leq p\right)^{T}$ denote the $j$-observation. Let $X_{n}=$ $\left(x_{i j}\right)$ be a $p \times n$ data matrix and $\hat{\rho}_{i j}$ be the sample correlation coefficient between the $i$ th and $j$ th rows of $X_{n}$. That is,

$$
\hat{\rho}_{i j}=\frac{\sum_{k=1}^{n}\left(x_{i k}-\bar{x}_{i}\right)\left(x_{j k}-\bar{x}_{j}\right)}{\sqrt{\sum_{k=1}^{n}\left(x_{i k}-\bar{x}_{i}\right)^{2}} \sqrt{\sum_{k=1}^{n} \sum_{k=1}^{n}\left(x_{j k}-\bar{x}_{j}\right)^{2}}}
$$

where $\bar{x}_{i}=(1 / n) \sum_{k=1}^{n} x_{i k}$. Then, $R_{n}:=\left(\hat{\rho}_{i j}\right)$ is the $p \times p$ sample correlation matrix generated by $X_{n}$. Let $\omega_{i j}=(1 / n) \sum_{k=1}^{n}\left(x_{i k}-\bar{x}_{i}\right)\left(x_{j k}-\bar{x}_{j}\right)$; then, the sample covariance matrix is defined as $S_{n}=\left(\omega_{i j}\right)$. Let $T_{n}$ and $L_{n}$ denote the largest entries of off-diagonal elements of $S_{n}$ and $R_{n}$, respectively, that is,

$$
\begin{aligned}
& T_{n}=\max _{1 \leq i<j \leq p}\left|\omega_{i j}\right|, \\
& L_{n}=\max _{1 \leq i<j \leq p}\left|\widehat{\rho}_{i j}\right| .
\end{aligned}
$$

In this paper, we mainly study the asymptotic properties of $T_{n}, L_{n}$. Assume that

(i) $p$ and $n$ both tend to infinity and $(p / n) \longrightarrow y \in(0, \infty)$

(ii) For each $1 \leq j \leq n$, the component of $\mathbf{x}_{j}=\left(x_{i j}, 1 \leq i \leq p\right)^{T}$ are independent

(iii) $E x_{i k}=0$ and $E x_{i k}^{2}=1$, for all $1 \leq i \leq p, 1 \leq k \leq n$, and $\sup _{i, k} E\left|x_{i k}\right|^{30+\tau}<\infty$, for some $\tau>0$

Theorem 1. Under above assumptions (i)-(iii), we have that

$$
\lim _{n \rightarrow \infty} \sqrt{\frac{n}{\log n}} T_{n}=2 \quad \text { a.s. }
$$

Theorem 2. Under above assumptions (i)-(iii), we have that

$$
P\left(n T_{n}^{2}-a_{n} \leq t\right) \longrightarrow \exp \left\{-\frac{1}{\sqrt{8 \pi}} e^{-(t / 2)}\right\},
$$

as $n \longrightarrow \infty$, for any $t \in R$, where $a_{n}=4 \log p+\log (\log p)$.

The limiting distribution appearing in Theorem 1 is called the extreme distribution of type I. Jiang [6] has proved that Theorems 1 and 2 hold if $x_{i k}$ are i.i.d. random variables under some moment conditions.

Theorem 3. Under above assumptions (i)-(iii), we have that

$$
\lim _{n \longrightarrow \infty} \sqrt{\frac{n}{\log n}} L_{n}=2 \quad \text { a.s.. }
$$

Theorem 4. Under above assumptions (i)-(iii), we have that

$$
P\left(n L_{n}^{2}-a_{n} \leq t\right) \longrightarrow \exp \left\{-\frac{1}{\sqrt{8 \pi}} e^{-t / 2}\right\}
$$

as $n \longrightarrow \infty$, for any $t \in R$, where $a_{n}=4 \log p+\log (\log p)$.

Remark 1. Note that $\mathbf{x}_{1}, \mathbf{x}_{2}, \ldots, \mathbf{x}_{n}$ are $n$ independent observations of the $p$-dimensional real-valued random vector $\mathbf{x}$; therefore, they are independent and identically distributed random vectors.

Remark 2. Our proofs rely on Lemma 1; therefore, the moment condition may be relaxed if the convergence rate in Lemma 1 can be improved; this question will be left for later.

The statistics inference for ultra-high-dimensional data have been studied a lot these years because of its wide applications in many areas. In these applications, the dimension $p$ can be much larger than the sample size $n$; it may happen that $(p / n) \longrightarrow \infty$. Cai and Jiang [15] investigate the limit properties of $L_{n}$ for $p \gg n$ for i.i.d. random variables under more stronger moment assumption. It is interesting problem that can the identically distributed assumption be removes in the setting $(p / n) \longrightarrow \infty$. We will investigate this topic in the future.

\section{Proof of Main Results}

We first introduce some lemmas that are used for the proofs of main results. Let $\eta_{1}, \ldots, \eta_{n}$ be independent random variables such that $E \eta_{j}=0, E \eta_{j}^{2}=\sigma_{j}^{2}, E\left|\eta_{j}\right|^{k}<\infty, j=$ $1,2, \ldots, n$, for integer $k \geq 3$. Denote $f_{j}$ and $\gamma_{v j}$ the characteristic function and $v$-order cumulant of $\eta_{j}$, respectively. Let $B_{n}^{2}=\sum_{j=1}^{n} \sigma_{j}^{2}$ and $F_{n}$ be the distribution function of $B_{n}^{-1} \sum_{j=1}^{n} \eta_{j}$. Set

$$
\begin{gathered}
\lambda_{\nu, n}=n^{(\nu-2) / 2} B_{n}^{-\nu} \sum_{j=1}^{n} \gamma_{\nu j}, \quad v=3,4, \ldots, k, \\
Q_{\nu, n}(x)=\sum^{\prime}(-1)^{\nu+2 s} \Phi^{(\nu+2 s)}(x) \prod_{m=1}^{\nu} \frac{1}{k_{m} !}\left(\frac{\lambda_{m+2, n}}{(m+2) !}\right)^{k_{m}},
\end{gathered}
$$

where $\Phi(x)$ is distribution function of standard normal distribution and the summation $\sum$ is taken over all nonnegative integer solutions $\left(k_{1}, k_{2}, \ldots, k_{v}\right)$ of the equations:

$$
\begin{aligned}
& k_{1}+k_{2}+\cdots+k_{v}=s, \\
& k_{1}+2 k_{2}+\cdots+v k_{v}=v .
\end{aligned}
$$

For each real $x$, let

$$
\begin{aligned}
Y_{n j} & =\eta_{j} I\left(\left|\eta_{j}\right| \leq B_{n}\right), \\
Z_{n j}^{(x)} & =\eta_{j} I\left(\left|\eta_{j}\right| \leq B_{n}(1+|x|)\right), \\
W_{n j}^{(x)} & =\eta_{j} I\left(\left|\eta_{j}\right|>B_{n}(1+|x|)\right), \quad j=1,2, \ldots, n .
\end{aligned}
$$


Lemma 1 (see Theorem 1 in [16]). Suppose that $\eta_{1}, \ldots, \eta_{n}$ are independent random variables such that
$E \eta_{j}=0, E \eta_{j}^{2}=\sigma_{j}^{2}, E\left|\eta_{j}\right|^{k}<\infty, j=1,2, \ldots, n$, for integer $k \geq 3$. Let $B_{n}^{2}=\sum_{j=1}^{n} \sigma_{j}^{2}$. Then, we have

$$
\begin{aligned}
& \left|F_{n}(x)-\Phi(x)-\sum_{v=1}^{k-1} Q_{\gamma, n}(x) n^{-(1 / 2)}\right| \\
\leq & C(k)\left\{(1+|x|)^{-k} B_{n}^{-k} \sum_{j=1}^{n} E\left|W_{n}^{(x)}\right|^{k}+(1+|x|)^{-k-1} B_{n}^{-k-1} \times \sum_{j=1}^{n} E\left|Z_{n}^{(x)}\right|^{k+1}\right. \\
+ & \left.(1+|x|)^{-k-1} n^{(1 / 2) k(k+1)} \times\left(\sup _{|t| \geq \delta_{n}} \frac{1}{n} \sum_{j=1}^{n}\left|f_{j}(t)\right|+\frac{1}{2 n}\right)^{n}\right\},
\end{aligned}
$$

where $\delta_{n}=(1 / 12) B_{n}^{2}\left(\sum_{j=1}^{n} E\left|Y_{n j}\right|^{3}\right)^{-1}$ and $C(k)$ means $a b$ solute constants only depending on $k$.

Lemma 2 (see Lemma A.1 in [6]). If $\left\{\eta_{n}, n \geq 1\right\}$ are i.i.d. random variables with $E \eta_{1}=0, E\left|\eta_{1}\right|^{p}<\infty, \quad p \geq 1$, and $S_{n}=\sum_{i=1}^{n} \eta_{i}$, then

$$
E\left|S_{n}\right|^{p}= \begin{cases}O\left(n^{p / 2}\right), & \text { if } p \geq 2, \\ O(n), & \text { if } 1 \leq p<2 .\end{cases}
$$

Lemma 3 (see Lemma A.2 in [6]). Let $\left\{\eta_{k}, 1 \leq k \leq n\right\}$ be independent symmetric random variables and $S_{n}=\sum_{k=1}^{n} \eta_{k}$. Then, for each integer $j \geq 1$, there exist positive numbers $C_{j}$ and $D_{j}$ depending only on $j$ such that, for all $t>0$,

$$
P\left(\left|S_{n}\right| \geq 2 j t\right) \leq C_{j} P\left(\max _{1 \leq k \leq n}\left|\eta_{j}\right| \geq t\right)+D_{j}\left(P\left(\left|S_{n}\right| \geq t\right)\right)^{j} .
$$

Lemma 4 (see Lemma A.4 in [6]). Let I be an index set and $\left\{B_{\alpha}, \alpha \in I\right\}$ be a set of subsets of $I$; that is, $B_{\alpha} \subset I$, for each $\alpha \in I$. Let also $\left\{\eta_{\alpha}, \alpha \in I\right\}$ be random variables. For a given $t \in \mathbb{R}$, set $\lambda=\sum_{\alpha \in I} P\left(\eta_{\alpha}>t\right)$. Then,

$$
\left|P\left(\max _{\alpha \in I} \eta_{\alpha} \leq t\right)-e^{-\lambda}\right| \leq\left(1 \wedge \lambda^{-1}\right)\left(b_{1}+b_{2}+b_{3}\right),
$$

where

$$
\begin{aligned}
b_{1} & =\sum_{\alpha \in I} \sum_{\beta \in B_{\alpha}} P\left(\eta_{\alpha}>t\right) P\left(\eta_{\beta}>t\right), \\
b_{2} & =\sum_{\alpha \in I} \sum_{\alpha \neq \beta \in B_{\alpha}} P\left(\eta_{\alpha}>t, \eta_{\beta}>t\right), \\
b_{3} & \sum_{\alpha \in I} E\left|P\left(\eta_{\alpha}>t \mid \sigma\left(\eta_{\beta}, \beta \notin B_{\alpha}\right)\right)-P\left(\eta_{\alpha}>t\right)\right|,
\end{aligned}
$$

and $\sigma\left(\eta_{\beta}, \beta \notin B_{\alpha}\right)$ is the $\sigma$-algebra generated by $\left\{\eta_{\beta}, \beta \notin B_{\alpha}\right\}$. In particular, if $\eta_{\alpha}$ is independent of $\left\{\eta_{\beta}, \beta \notin B_{\alpha}\right\}$ for each $\alpha$, then $b_{3}=0$.
Lemma 5 (see Theorem 3 in [6]). Let $\left\{\eta_{i}, 1 \leq i \leq n\right\}$ be independent random variables with mean zero. Assume $\max _{1 \leq i \leq n} E\left|\eta_{i}\right|^{\beta}<\infty$, for some $\beta>2$. Then, for any $\rho>0$ and $t>0$,

$$
P\left(\frac{1}{\sqrt{n}} \sum_{i=1}^{n} \eta_{i} \geq t\right) \leq \frac{M_{\beta}}{n^{\rho \beta-1}}+K_{n} e^{-t_{\rho}^{2} /\left(2 M_{2}\right)},
$$

where

$$
\begin{aligned}
& M_{s}:=\frac{1}{n} \sum_{i=1}^{n} E\left|\eta_{i}\right|^{s} \quad \text { for } s \in(0, \beta], \\
& K_{n}:=\exp \left\{\frac{t^{3} n^{\rho-(1 / 2)}}{3 M_{2}^{2}} e^{\left(2 t n^{\rho-(1 / 2)}\right) / M_{2}}\right\},
\end{aligned}
$$

and $t^{\rho}=t-M_{\beta} n^{\rho(1-\beta)+(1 / 2)}$.

Now, we investigate the limit properties of $W_{n}$ defined as follows:

$$
W_{n}=\frac{1}{n} \times \max _{1 \leq i<j \leq p}\left|\sum_{k=1}^{n} x_{i k} x_{j k}\right|
$$

Proposition 1. Under above assumptions (i)-(iii), we have that

$$
\lim _{n \longrightarrow \infty} \sqrt{\frac{n}{\log n}} W_{n}=2 \quad \text { a.s.. }
$$

Proposition 2. Under above assumptions (i)-(iii), we have that

$$
P\left(n W_{n}^{2}-a_{n} \leq t\right) \longrightarrow \exp \left\{-\frac{1}{\sqrt{8 \pi}} e^{-t / 2}\right\},
$$

as $n \longrightarrow \infty$, for any $t \in R$, where $a_{n}=4 \log p+\log (\log p)$.

Proof. of Proposition 1. We only need to prove that 


$$
\begin{aligned}
& \limsup _{n \longrightarrow \infty} \sqrt{\frac{n}{\log n}} W_{n} \leq 2 \quad \text { a.s., } \\
& \liminf _{n \longrightarrow \infty} \sqrt{\frac{n}{\log n}} W_{n} \geq 2 \quad \text { a.s.. }
\end{aligned}
$$

In order to prove (20), we only need to prove that, for any $\delta>0$,

$$
\limsup _{n \rightarrow \infty} \sqrt{\frac{n}{\log n}} W_{n} \leq 2+\delta \quad \text { a.s.. }
$$

Let $m$ be a integer satisfying that $m \delta>4$; then, we have, for sufficient large integer $d$,

$$
\begin{aligned}
& P\left(\sqrt{\frac{d^{m}}{\log d^{m}}} W_{d^{m}}>2+\delta\right) \\
= & P\left(\max _{1 \leq i<j \leq p\left(d^{m}\right)}\left|\frac{1}{\sqrt{d^{m}}} \sum_{k=1}^{d^{m}} x_{i k} x_{j k}\right|>(2+\delta) \sqrt{\log d^{m}}\right) \\
\leq & 4 y d^{2 m} \max _{1 \leq i<j \leq p\left(d^{m}\right)} P\left(\left|\frac{1}{\sqrt{d^{m}}} \sum_{k=1}^{d^{m}} x_{i k} x_{j k}\right|>(2+\delta) \sqrt{\log d^{m}}\right) .
\end{aligned}
$$

By Lemma 1, we have that

$$
\max _{1 \leq i<j \leq p\left(d^{m}\right)} P\left(\frac{1}{\sqrt{d^{m}}}\left|\sum_{k=1}^{d^{m}} x_{i k} x_{j k}\right|>(2+\delta) \sqrt{\log d^{m}}\right)=O\left(\frac{1}{d^{m(2+\delta)}}\right) .
$$

Thus, we have that $\sum_{d=1}^{\infty} P\left(\sqrt{\left(d^{m} / \log d^{m}\right)} W_{d^{m}}\right.$ $>2+\delta)<\infty$. Then, by Borel-Cantelli lemma, we conclude that

$$
\limsup _{d \rightarrow \infty} \sqrt{\frac{d^{m}}{\log d^{m}}} W_{d^{m}} \leq 2+\delta \quad \text { a.s.. }
$$

For $d^{m} \leq n<(d+1)^{m}$, we have that

$$
\max _{d^{m} \leq n<(d+1)^{m}}\left|n W_{n}-d^{m} W_{d^{m}}\right| \leq \max _{d^{m} \leq n<(d+1)^{m}} \max _{1 \leq i<j \leq(d+1)^{m}}\left|\sum_{k=d^{m}+1}^{n} x_{i k} x_{j k}\right| .
$$

Note that

$$
\begin{aligned}
& P\left(\max _{d^{m} \leq n<(d+1)^{m}} \max _{1 \leq i<j \leq(d+1)^{m}}\left|\sum_{k=d^{m}+1}^{n} x_{i k} x_{j k}\right|>\delta \sqrt{d^{m} \log d^{m}}\right) \\
& \leq 2(d+1)^{2 m} \max _{1 \leq i<j \leq(d+1)^{m}} P\left(\max _{d^{m} \leq n<(d+1)^{m}}\left|\sum_{k=d^{m}+1}^{n} x_{i k} x_{j k}\right|>\delta \sqrt{d^{m} \log d^{m}}\right) .
\end{aligned}
$$


Mathematical Problems in Engineering

5

Then, by Ottaviani's inequality, we have that, for sufficlient large $d$,

$$
\begin{aligned}
& P\left(\max _{d^{m} \leq n<(d+1)^{m}}\left|\sum_{k=d^{m}+1}^{n} x_{i k} x_{j k}\right| \geq \delta \sqrt{d^{m} \log d^{m}}\right) \\
\leq & P\left(\left|\sum_{k=d^{m}+1}^{x_{j k}^{m}} x_{i k} x_{j k}\right| \geq\left(\delta \sqrt{d^{m} \log d^{m}} / 2\right)\right) \\
\min _{d^{m} \leq n<(d+1)^{m}} P\left(\left|\sum_{k=n+1}^{x_{j k}^{m}} x_{i k} x_{j k}\right| \leq\left(\delta \sqrt{d^{m} \log d^{m}} / 2\right)\right) & \\
\leq & 2 P\left(\left|\sum_{k=d^{m}+1}^{x_{j k}^{m}} x_{i k} x_{j k}\right| \geq\left(\delta \frac{\sqrt{d^{m} \log d^{m}}}{2}\right)\right) .
\end{aligned}
$$

By Lemma 1, we know that, for sufficient large $d$,

$$
P\left(\left|\sum_{k=d^{m}+1}^{(d+1)^{m}} x_{i k} x_{j k}\right| \geq \delta \frac{\sqrt{d^{m} \log d^{m}}}{2}\right)=O\left(\frac{1}{d^{4 m}}\right) \text {. }
$$

Then,

$$
P\left(\max _{d^{m} \leq n<(d+1)^{m}} \max _{1 \leq i<j \leq(d+1)^{m}}\left|\sum_{k=d^{m}+1}^{n} x_{i k} x_{j k}\right|>\delta \sqrt{d^{m} \log d^{m}}\right)=O\left(\frac{1}{d^{2 m}}\right)
$$

is summable. Noticing the fact that $\left(\sqrt{(d+1)^{m} \log (d+1)^{m}} / \sqrt{d^{m} \log d^{m}}\right) \longrightarrow 1$, we conclude that

$$
\limsup _{d \rightarrow \infty} \max _{d^{m} \leq n \leq(d+1)^{m}}\left|\sqrt{\frac{d^{m}}{\log d^{m}}} W_{d^{m}}-\sqrt{\frac{n}{\log n}} W_{n}\right| \leq \delta, \quad \text { ass.. }
$$

Since $\delta$ is arbitrary, combining with (25), we know that (20) is true.

Now, we prove (21). In Lemma 4, take $I=\{(i, j) ; 1 \leq i<j \leq p\}$. For $\alpha=(i, j) \in I$, set $B_{\alpha}=\{(k, l)$ $\in I$; one of $k$ and $l=i$ or $j$ but $(k, l) \neq \alpha\}, \quad \eta_{\alpha}=\left|\sum_{k=1}^{n} x_{i k} x_{j k}\right|$, $t=(2-\delta) \sqrt{n \log n}$. Note that $\eta_{\alpha}$ is independent of $\left\{\eta_{\beta}, \beta \notin B_{\alpha}\right\}$, and we have

$$
P\left(n W_{n} \leq t\right)=P\left(\max _{\alpha \in I} \eta_{\alpha} \leq t\right) \leq e^{-\lambda_{n}}+b_{1, n}+b_{2, n} .
$$

Here,

$$
\begin{aligned}
\lambda_{n} & =\sum_{\alpha \in I} P\left(\left|\eta_{\alpha}\right|>t\right)=\sum_{1 \leq i<j \leq p} P\left(\left|\sum_{k=1}^{n} x_{i k} x_{j k}\right|>(2-\delta) \sqrt{n \log n}\right) \\
b_{1, n} & =\sum_{\alpha \in I} \sum_{\beta \in B_{\alpha}} P\left(\eta_{\alpha}>t\right) P\left(\eta_{\beta}>t\right) \\
& \leq 2 p^{3} \max _{1 \leq i<j \leq p} P^{2}\left(\left|\sum_{k=1}^{n} x_{i k} x_{j k}\right|>(2-\delta) \sqrt{n \log n}\right) \\
b_{2, n} & =\sum_{\alpha \in I} \sum_{\alpha \neq \beta \in B_{\alpha}} P\left(\eta_{\alpha}>t, \eta_{\beta}>t\right) \\
& \leq 2 p^{3} \max _{1 \leq i<j \leq p, 1 \leq l \neq j \leq p} P\left(\left|\sum_{k=1}^{n} x_{i k} x_{j k}\right|>(2-\delta) \sqrt{n \log n}\right. \\
& \left.\left|\sum_{k=1}^{n} x_{i k} x_{l k}\right|>(2-\delta) \sqrt{n \log n}\right) .
\end{aligned}
$$

Remember that $\left\{x_{i k} x_{j k}\right\}$ is a sequence of independent random variables with mean 0 and variance 1 . From the moment assumption (iii), by Lemma 1, we know that, for sufficiently large $n$,

$$
\left|P\left(\left|\sum_{k=1}^{n} x_{i k} x_{j k}\right|>(2-\delta) \sqrt{n \log n}\right)-\frac{1}{(2-\delta) \sqrt{2 \pi \log n}} \cdot \frac{1}{n^{(2-\delta)^{2} / 2}}\right| \leq \frac{1}{n^{2-\delta^{\prime}}},
$$


where $1>\delta^{\prime}>\delta$ is a constant. Combining with later Lemma 6 , we obtain that, for sufficiently large $n$,

$$
\begin{aligned}
e^{-\lambda_{n}} & =O\left(e^{-n^{\delta^{\prime}}}\right), \\
b_{1, n} & =O\left(\frac{1}{\sqrt{n}}\right), \\
b_{2, n} & =O\left(\frac{1}{\sqrt{n}}\right) .
\end{aligned}
$$

Then, from (32) and (35), we conclude that there is a $\varepsilon>0$ depending on $\delta$ such that

$$
P\left(n W_{n} \leq(2-\delta) \sqrt{n \log n}\right)=O\left(\frac{1}{n^{\epsilon}}\right) .
$$

Choose an integer $m$ such that $m \varepsilon>1$. Then, $P\left(d^{m} W_{d^{m}} \leq(2-\delta) \sqrt{d^{m} \log d^{m}}\right)=O\left(1 / d^{m \varepsilon}\right)$. Since $\sum_{d}$ $d^{-m \varepsilon}<\infty$, by the Borel-Cantelli lemma, we have that

$$
\liminf _{d \rightarrow \infty} \sqrt{\frac{d^{m}}{\log \left(d^{m}\right)}} W_{d^{m}} \geq 2-\delta \quad \text { a.s. }
$$

for any $\delta \in(0,1)$. Note that, for $d^{m} \leq n<(d+1)^{m}$,

$$
\inf _{d^{m} \leq n<(d+1)^{m}} n W_{n} \geq d^{m} W_{d^{m}}-\max _{d^{m} \leq n<(d+1)^{m}} \max _{1 \leq i<j \leq p\left((d+1)^{m}\right)}\left|\sum_{k=d^{m}+1}^{n} x_{i k} x_{j k}\right| .
$$

By (30) and (37) and the fact that $\left(\sqrt{(d+1)^{m} \log (d+1)^{m}} / \sqrt{d^{m} \log d^{m}}\right) \longrightarrow 1$, we have that (21) holds.

Proof. of Proposition 2. Note that

$$
P\left(n W_{n}^{2}-a_{n} \leq t\right)=P\left(\max _{1 \leq i<j \leq p}\left|\sum_{k=1}^{n} x_{i k} x_{j k}\right| \leq \sqrt{n\left(t+a_{n}\right)}\right) .
$$

Now, we apply Lemma 4 to prove this proposition. Take $I=\{(i, j) ; 1 \leq i<j \leq p\}$, for $\alpha=(i, j) \in I$; set $X_{\alpha}=\mid \sum_{k=1}^{n}$ $x_{i k} x_{j k} \mid / \sqrt{n}$ and $B_{\alpha}=\{(k, l) \in I$; one of $k$ and $l=i$ or $j$, but $(k, l) \neq \alpha\}$. Define

$$
\lambda_{n}=\sum_{1 \leq i<j \leq p} P\left(X_{\alpha}>\sqrt{t+a_{n}}\right) .
$$

Note the sequence $\left\{x_{i k} x_{j k}\right\}$ is a sequence of independent random variables with mean 0 and variance 1 . It follows from Lemma 1 that, for $1 \leq i, j \leq p$,

$$
\begin{aligned}
P\left(X_{\alpha}>\sqrt{t+a_{n}}\right) & =P\left(\frac{1}{\sqrt{n}}\left|\sum_{k=1}^{n} x_{i k} x_{j k}\right|>\sqrt{t+a_{n}}\right) \\
& =\frac{e^{-t / 2}}{\sqrt{t+a_{n}} \sqrt{2 \pi}} p^{-2}+O\left(\frac{1}{p^{2+\epsilon}}\right),
\end{aligned}
$$

for some constant $\varepsilon>0$. Thus, it follows that

$$
\lambda_{n} \longrightarrow \frac{e^{-t / 2}}{\sqrt{8 \pi}}
$$

Observe that $X_{\alpha}$ is independent of $\left\{X_{\beta} ; \beta \in I \backslash B_{\alpha}\right\}$, for any $\alpha=(i, j) \in I$. Define that

$$
\begin{aligned}
& b_{1 n}=\sum_{\alpha \in I} \sum_{\beta \in B_{\alpha}} P\left(X_{\alpha}>\sqrt{t+a_{n}}\right) P\left(X_{\beta}>\sqrt{t+a_{n}}\right) \\
& b_{2 n} \sum_{\alpha \in I} \sum_{\alpha \neq \beta \in B_{\alpha}} P\left(X_{\alpha}>\sqrt{t+a_{n}}, X_{\beta}>\sqrt{t+a_{n}}\right) .
\end{aligned}
$$

It can be seen that there at most $2 p$ terms in $B_{\alpha}$. Thus, by (41), we have

$$
b_{1 n} \leq \frac{1}{2}\left(p^{2}-p\right) \cdot(2 p) \cdot \max _{1 \leq i<j \leq p} P^{2}\left(X_{\alpha}>\sqrt{t+a_{n}}\right)=O\left(\frac{1}{p}\right) .
$$

Similarly,

$$
\begin{aligned}
& b_{2 n} \leq p\left(p^{2}-p\right) \times \\
& \max _{1 \leq i<j \leq p, 1 \leq l \neq j \leq n} P\left(\left|\sum_{k=1}^{n} x_{i k} x_{j k}\right|>\sqrt{n\left(t+a_{n}\right)},\left|\sum_{k=1}^{n} x_{i k} x_{l k}\right|>\sqrt{n\left(t+a_{n}\right)}\right) .
\end{aligned}
$$


By Lemma 6, we obtain that $b_{2 n}=O\left(n^{-b}\right)$ for some constant $b>0$ under the moment assumption. Then, by Lemma 4, this proposition is concluded.

Proof. of Theorems 1 and 2. Note that

$$
\left|\sum_{k=1}^{n}\left(x_{i k}-\bar{x}_{i}\right)\left(x_{j k}-\bar{x}_{j}\right)-\sum_{k=1}^{n} x_{i k} x_{j k}\right| \leq n \bar{x}_{i} \bar{x}_{j} .
$$

Therefore,

$$
\left|W_{n}-T_{n}\right| \leq \max _{i \neq j}\left|\bar{x}_{i} \bar{x}_{j}\right| \leq\left(\max _{1 \leq i \leq p}\left|\bar{x}_{i}\right|\right)^{2}
$$

Now, we estimate the last term in the above equation: $P\left(n^{1 / 4}(\log n)^{1 / 4} \max _{1 \leq i \leq p}\left|\bar{x}_{i}\right| \geq \epsilon\right) \leq p \times \max _{1 \leq i \leq p} P\left(n^{1 / 4}(\log n)^{1 / 4}\left|\bar{x}_{i}\right| \geq \epsilon\right)$.

By Lemma 1, we have that, for every $i$,

Thus,

$$
\begin{aligned}
\max _{1 \leq i \leq p} P\left(n^{1 / 4}(\log n)^{1 / 4}\left|\bar{x}_{i}\right| \geq \epsilon\right)=P\left(\frac{1}{\sqrt{n}}\left|\sum_{k=1}^{n} x_{i k}\right| \geq \epsilon n^{1 / 4}(\log n)^{-1 / 4}\right) \leq C n^{-2} . \\
n\left(W_{n}^{2}-T_{n}^{2}\right)=n\left(W_{n}-T_{n}\right)\left(W_{n}+T_{n}\right) \longrightarrow 0, \quad \text { a.s.. }
\end{aligned}
$$

$$
\sqrt{n \log n}\left(\max _{1 \leq i \leq p}\left|\bar{x}_{i}\right|\right)^{2)} \longrightarrow 0, \quad \text { a.s. }
$$

Combining with (47), we have that

$$
\sqrt{n \log n}\left|W_{n}-T_{n}\right| \longrightarrow 0 \text {, a.s.. }
$$

Then, Theorem 1 follows. From above equation and Proposition 1, we know that $W_{n}+T_{n}=O\left((\log n)^{1 / 2} n^{-1 / 2}\right)$. Applying (47) and (50), we obtain that

\section{Theorem 2 then follows.}

Proof. of Theorems 3 and 4. Under conditions (i)-(iii), we have from the triangle inequality that

$$
\begin{aligned}
\left|L_{n}-T_{n}\right| & \leq \max _{1 \leq i<j \leq p}\left|\hat{\rho}_{i j}-\omega_{i j}\right| \\
& \leq T_{n} \max _{1 \leq i<j \leq p}\left|1-\frac{\sqrt{n}}{\sqrt{\sum_{k=1}^{n}\left(x_{i k}-\bar{x}_{i}\right)^{2}}} \frac{\sqrt{n}}{\sqrt{\sum_{k=1}^{n}\left(x_{j k}-\bar{x}_{j}\right)^{2}}}\right| \\
& \leq T_{n} \times D_{1}^{-2}\left(D_{2}^{2}+2 D_{2}\right),
\end{aligned}
$$

where

$$
\begin{aligned}
& D_{1}=\min _{1 \leq i \leq p} \frac{\sqrt{\sum_{k=1}^{n}\left(x_{i k}-\bar{x}_{i}\right)^{2}}}{\sqrt{n}}, \\
& D_{2}=\max _{1 \leq i \leq p}\left|\frac{\sqrt{\sum_{k=1}^{n}\left(x_{i k}-\bar{x}_{i}\right)^{2}}}{\sqrt{n}}-1\right| .
\end{aligned}
$$

From the fact that $|x-1| \leq\left|x^{2}-1\right|$, for any $x>0$, we have that

$$
\begin{aligned}
& P\left(\log n \times \max _{1 \leq i \leq p}\left|\frac{\sum_{k=1}^{n} x_{i k}^{2}-n}{n}\right| \geq \epsilon\right) \\
& \leq p \times \max _{1 \leq i \leq p} P\left(\frac{1}{\sqrt{n}}\left|\sum_{k=1}^{n} x_{i k}^{2}-n\right| \geq \epsilon n^{1 / 2}(\log n)^{-1}\right) \leq n^{-2} .
\end{aligned}
$$

We can see that the sum above is summable. Similarly, we have that

$$
\begin{aligned}
& P\left(\sqrt{\log n} \max _{1 \leq i \leq p}\left|\bar{x}_{i}\right| \geq \epsilon\right) \\
& \leq p \times \max _{1 \leq i \leq p} P\left(\frac{1}{\sqrt{n}}\left|\sum_{k=1}^{n} x_{i k}\right| \geq \epsilon n^{1 / 2}(\log n)^{-1 / 2}\right)=O\left(n^{-2}\right) .
\end{aligned}
$$


It is also summable. Thus, we conclude that $(\log n) \times$ $D_{2} \longrightarrow 0$ almost surely. It can be seen from above discussion that $D_{1} \longrightarrow 1$ almost surely. Combining these facts with Theorem 1, we have that

$$
\begin{array}{r}
\sqrt{\frac{n}{\log n}}\left|L_{n}-T_{n}\right| \longrightarrow 0, \quad \text { a.s. } \\
n\left(L_{n}^{2}-T_{n}^{2}\right)=n\left(L_{n}-T_{n}\right)\left(L_{n}+T_{n}\right) \longrightarrow 0, \quad \text { a.s.. }
\end{array}
$$

Theorems 3 and 4 then follow.

Lemma 6. Let $v_{n}=\sqrt{n\left(t+a_{n}\right)}$ and $u_{n}=(2-\delta) \sqrt{n \log n}$ be defined before. Then, we have, for some constant $b>0$,

$$
\begin{aligned}
& \max _{1 \leq i<j \leq p, 1 \leq l \neq i \leq p} P\left(\left|\sum_{k=1}^{n} x_{i k} x_{j k}\right|>v_{n},\left|\sum_{k=1}^{n} x_{i k} x_{l k}\right|>v_{n}\right)=O\left(n^{-3-b}\right), \\
& \max _{1 \leq i<j \leq p, 1 \leq l \neq i \leq p} P\left(\left|\sum_{k=1}^{n} x_{i k} x_{j k}\right|>u_{n},\left|\sum_{k=1}^{n} x_{i k} x_{l k}\right|>u_{n}\right)=O\left(n^{-7 / 2}\right),
\end{aligned}
$$

as $n \longrightarrow \infty$.

Proof. Note that, for each $i$, the sequence $\left\{x_{i k}\right\}$ is independent identically distributed random variables and $\left\{x_{i k}\right\},\left\{x_{i k}\right\}$, and $\left\{x_{l k}\right\}$ are independent. With the moment assumption (iii), the conclusion can be obtained by modifying Lemma 2.1 in [6].

\section{Data Availability}

No data were used to support the findings of this study.

\section{Conflicts of Interest}

The author declares that they have no conflicts of interest.

\section{Acknowledgments}

This study was supported by Science and Technology Program of Jilin Educational Department during the "13th FiveYear" Plan Period (Grant no. JJKH20200951KJ) and National Science Foundation of China (Grant no. 12171198).

\section{References}

[1] Z. Bai, J. Silverstein, Spectral analysis of large dimensional random matrices," Springer Series in Statistics, Springer, New York, NY, USA, second edition, 2010.

[2] J. Yao, S. Zheng, and Z. Bai, Large Sample Covariance Matrices and High Dimensional Data Analysis, Cambridge University Press, Cambridge, UK, 2015.

[3] T. Jiang, "The limiting distributions of eigenvalues of sample correlation matrices," Sankhya, vol. 66, pp. 35-48, 2004.

[4] J. Gao, X. Han, G. Pan, and Y. Yang, "High dimensional correlation matrices: the central limit theorem and its applications," Journal of the Royal Statistical Society: Series B, vol. 79, no. 3, pp. 677-693, 2017.

[5] X. Mestre and P. Vallet, "Correlation tests and linear spectral statistics of the sample correlation matrix," IEEE Transactions on Information Theory, vol. 63, no. 7, pp. 4585-4618, 2017.
[6] T. Jiang, "The asymptotic distribution of the largest entries of sample correlation matrices," Annals of Applied Probability, vol. 14, pp. 865-880, 2004.

[7] D. Li and A. Rosalsky, "Some strong limit theorems for the largest entries of sample correlation matrices," Annals of Applied Probability, vol. 16, pp. 423-447, 2006.

[8] D. Li, W.-D. Liu, and A. Rosalsky, "Necessary and sufficient conditions for the asymptotic distribution of the largest entry of a sample correlation matrix," Probability Theory and Related Fields, vol. 148, no. 1-2, pp. 5-35, 2010.

[9] D. Li, Y. Qi, and A. Rosalsky, "On Jiang's asymptotic distribution of the largest entry of a sample correlation matrix," Journal of Multivariate Analysis, vol. 111, pp. 256-270, 2012.

[10] D. Li and L. Xue, "Joint limiting laws for high dimensional independence tests," arXiv:1512.08819V1, 2015.

[11] D. Li, L. Xue, and H. Zou, "Applications of peter hall's martingale limit theory to estimating and testing high dimensional covaraince matrices," Statistica Sinica, vol. 28, no. 4, pp. 2657-2670, 2018.

[12] X. Yu, D. Li, and L. Xue, "Fisher's combined probability test for high-dimensional covariance matrices," arXiv: 2006.00426, 2020.

[13] S. Zheng, G. Cheng, J. Guo, and H. Zhu, "Test for high-dimensional correlation matrices," Annals of Statistics, vol. 47, no. 5, pp. 2887-2921, 2019.

[14] W. Liu and Z. Lin, "Asymptotic distributions of the largest entries of sample correlation matrices under dependence assumptions," Chinese Annals of Mathamatics Series A, vol. 29, no. 3, pp. 309-324, 2008.

[15] T. Cai and T. Jiang, "Limiting laws of coherence of random matrices with applications to testing covariance structure and construction of compressed sensing matrices," Annals of Statistics, vol. 39, no. 3, p. 1496C1525, 2011.

[16] Z. Bai and L. Zhao, "Edgeworth expansions of distribution functions of independent random variables," Scientia SinicaSeries A, vol. 29, no. 1, pp. 1-22, 1986. 